stanischen Rechts läßt es die Mängel und Vorzüge der verschiedenen Rechtssysteme deutlich werden und bietet damit einen wertvollen Beitrag zur Lösung des Problems der richterlichen Kontrolle des Verwaltungshandelns. Bedauerlich ist, daß der Verfasser auf die kontinentaleuropäische Praxis kaum eingeht und deren System (z. B. eigene Verwaltungsgerichtsbarkeit) als Anregung für eine Lösung der Probleme in Indien und $\mathrm{Pa}$ kistan nicht diskutiert.

Henning v. Wedel

Peter Kunstadter (Hrsg.)

Southeast Asian Tribes, Minorities, and Nations

Princeton University Press, Princeton, N. J., 1967. XIII,

902 Seiten (2 Bände), $\$ 22,50$

Wie viele Wissenschaften, die sich in Deutschland mit überseeischen Regionen befassen, arbeitet auch die Völkerkunde bei uns weitgehend isoliert von den politischen Entwicklungen der Gegenwart und liefert kaum Erkenntnisse, die einen Beitrag zur Lösung der brennenden Probleme der "Dritten Welt" darstellen könnten. Völkerkunde in Deutschland ist noch vielfach die Beschäftigung mit Primitivkulturen, die als isolierte Gruppierungen behandelt werden, und in der Arbeit der Völkerkunde scheint oft ein Bedauern darüber mitzuschwingen, daß diese Primitivkulturen nicht mehr bereit sind, die Erfüllung ihres Lebenszweckes darin zu erblicken, als Beute „intellektueller Kopfjäger" zu dienen. So jedenfalls nennt der Herausgeber dieses Werkes jene Wissenschaftler, die nicht daran interessiert sind, den Gegenstand ihrer Forschung in einen größeren Zusammenhang hineinzustellen oder gar durch ihre Forschungen politische Entscheidungshilfen zu liefern.

Es mag aus diesem Grunde auch die $\mathrm{Ge}-$ fahr bestehen, daß der politisch interessierte Leser an dem Sammelwerk Kunstadters vorbeigeht, da er es für eine abseitige Spezialstudie halten könnte, in der von Animismus, Kopfjägerei und Fruchtbarkeitsriten die Re- de ist. Tatsächlich handelt es sich bei diesem Buch, mit dem die Ergebnisse einer Konferenz des Center of International Studies der Princeton University vom Mai 1965 vorgelegt werden, um einen hoch aktuellen Beitrag zur politischen Problematik der südostasiatischen Länder und zu ihren Bemühungen, Nationen zu werden.

Wer zunächst in den beiden Bänden des Werkes blättert, wird erstaunt sein über die Unzahl von Stämmen und Minderheiten, die in Südostasien leben und die nicht so kontaktlos sind, wie man denken mag, sondern die nicht selten einen entscheidenden Einfluß auf das Geschick ihrer Heimatländer gehabt haben oder noch besitzen.

Behandelt werden Birma, Laos, Malaysia, Thailand und Vietnam sowie die unmittelbar an Südostasien angrenzenden Gebiete Süd-Chinas und NordostIndiens. $\mathrm{Zu}$ jedem dieser Länder bzw. Gebiete wie auch zu dem gesamten Werk hat der Herausgeber Einführungen verfaßt, die mit Bibliographien und Übersichten über die jeweils anzutreffenden Stämme, die Anzahl ihrer Bevölkerungen, ihre Siedlungsgebiete und ihre sprachlichen Kennzeichnungen versehen sind. Darüber hinaus finden sich eine Reihe instruktiver Photos und fünfzehn Kartenskizzen.

Einige Fakten, auf die Kunstadter in seiner allgemeinen Einführung hinweist, ziehen sich durch fast alle Beiträge des Werkes hin. Die wichtigsten seien hier herausgestellt:

1. Die Grenzen der heutigen Staaten Südostasiens wurden von den Kolonialmächten ohne Rücksicht auf ethnische Verhältnisse gezogen mit der Folge, daß zahlreiche Stämme oder Minderheiten auf den Gebieten mehrerer Staaten wohnen. Dies bedeutet Verunsicherung der Grenzen und die Gefahr von Grenzkonflikten.

2. Die Regierungen der südostasiatischen Staaten, die oft nicht in der Lage sind, Kontrolle über die Stämme in den Bergen und Grenzgebieten auszuüben, interessieren sich erst seit jüngster Zeit für deren Probleme, ohne daß dieses Interesse immer mit der Formulierung 
einer Politik der Assimilierung oder Integration einhergeht. Dagegen haben es vor allem die kommunistischen Staaten des Raumes, nämlich China und NordVietnam sowie das kommunistisch beherrschte Gebiet von Laos, vestanden, Konzepte zu entwickeln, mit denen sie als Beschützer und Freunde der Minderheiten auftreten.

3. Das erwachte Interesse an den Minderheiten leidet an einer Uberbetonung strategischer Aspekte, womit die praktischen Maßnahmen oft zum Scheitern verurteilt sind. Die ökonomische Nutzung der Minderheitengebiete erfolgt allzuoft zum Vorteil der herrschenden Schichten; feudale Strukturen werden nicht konsequent genug bekämpft; angesichts abnehmender Sterberaten bemüht man sich zu wenig um die Schaffung neuer ökonomischer Entfaltungsmöglichkeiten oder um wirksame Maßnahmen der Familienplanung; Regierungsämter der einzelnen Staaten und ausländische Entwicklungsbehörden haben es bisher nicht verstanden, ihre verschiedenen Maßnahmen in gehöriger Weise zu koordinieren.

Im Falle Birmas (Beiträge von F. K. Lehman und Maran La Raw) zeigt sich besonders deutlich, wie die nationale Einheit gefährdet ist, wenn es nicht gelingt, das Verhältnis zwischen den Stämmen und Minderheiten einerseits und der regierenden Bevölkerung andererseits in Ordnung zu bringen. Hier zeigt sich aber auch, daß es angesichts der vielfältigen Minderheiten mit ihren spezifischen Eigenarten und den unterschiedlichen Beziehungen zu ihnen keine einheitliche Minderheitenpolitik geben kann. Eine Regierung, die allzu bereitwillig das westliche Konzept einer "Nation" oder "Gesellschaft" übernimmt, kommt damit besonders leicht in Gefahr, ihr Verhältnis zu den verschiedenen Volksgruppen zu verderben.

Die Volksrepublik China (Beitrag von Richard K. Diao) hat dagegen gezeigt, $\mathrm{da} ß$ in der Anerkennung des Gegensatzes zwischen den Minderheiten, die im Südwesten des Landes 20 Prozent der Bevölkerung ausmachen, und der chinesischen (Han-)Bevölkerung der erste An- satz einer Lösung liegt. Der Nationalregierung war es dagegen mit ihrer Politik der grundsätzlichen Gleichbehandlung aller Rassen innerhalb des Staates nicht gelungen, einer solchen Lösung näherzukommen. Wenn es auch das wahre Ziel der Volksregierung ist, die Kontrolle über die nationalen Minderheiten zu verstärken, wofür der Autor zahlreiche Belege liefert, so war man doch zu Konzessionen auf religiösem und kulturellem Gebiet bereit und hat durch gut koordinierte Programme der Forschung und Erziehung sowie der politischen und ökonomischen Entwicklung den Beweis erbracht, daß der Regierung und der Kommunistischen Partei Chinas das Schicksal der Minderheiten nicht gleichgültig ist.

Im Gegensatz wiederum zu anderen Staaten der behandelten Region wirken sich in Indien (Beitrag von Robbins Burling) die Überbleibsel des Kastenwesens besonders integrationshemmend aus. Denn wenn es anderswo führenden Vertretern der Minderheiten gelingt, $\mathrm{zu}$ angesehenen Positionen innerhalb der etablierten Gesellschaft aufzusteigen, können diese innerhalb der Hindu-Gesellschaft nur Eingang in die niedrigste $\mathrm{Ka}$ ste finden. Die Probleme, denen sich Indien im Nordosten gegenübersieht, sind damit nicht nur aus rassischen Gegensätzen erklärbar, sondern auch ein Ergebnis der Kastenstruktur. Der Autor dieses Beitrages zeigt auch, daß die Minderheiten dieses Gebietes politisch engagiert sind und daß es unter ihnen relativ wenige Analphabeten gibt, so daß es ihnen nicht einzuleuchten vermag, daß man sie offiziell als "rückständig" ansieht. Nach Meinung des Autors wäre das halbe Problem gelöst, wenn die Bewohner des Flachlandes aufhörten, sich für zivilisierter als die Bergstämme zu halten (S. 228). Bedenkenswert ist auch die Feststellung des Autors, daß man statt der Förderung isolierter Agrarentwicklung Verkehrswege bauen sollte, die die Minderheitengebiete in das Marktsystem des gesamten Landes einbeziehen (S. $227 \mathrm{f}$.).

Wie wichtig für die Stabilität des Staates eine klare und überzeugende Min- 
derheitenpolitik ist, zeigt sich im Falle von Laos (Beiträge von G. M. T. Osborn, G. Linwood Barney und J. Thomas Ward) allein schon in den Tatsachen, daß die Laoten in ihrem eigenen Staate fast eine Minderheit darstellen, daß die städtische und damit politisch aktive Bevölkerung mehrheitlich aus Nicht-Laoten besteht und daß viele der in Laos lebenden Völker durch sehr starke verwandte Gruppen auch jenseits der Landesgrenzen vertreten sind. Auch in Laos hat sich die kommunistisch gelenkte Pathet-Lao-Bewegung im Gegensatz zur königlichen Regierung immer an den Minderheiten interessiert gezeigt, was nicht ohne Einfluß auf die innenpolitische Entwicklung eines Landes bleiben kann, in welchem nach Beendigung der Feindseligkeiten diese Minderheiten unüberhörbar ihren Anspruch auf Mitwirkung an der politischen Verantwortung erheben werden. Peter Kunstadter und Joel Halpern weisen in diesem Zusammenhang besonders auf die sehr aktiven und politisch wachen Meo hin, von denen aber mehr Vertreter in Nord-Vietnam als in Laos leben (S. 249).

Die Beiträge über Malaysia beschränken sich auf die Verhältnisse in Sarawak (Tom Harrisson) und Sabah (C. H. Ley), aus denen sehr deutlich die Problematik des jungen Staates herausklingt, die auch Sukarno in seiner Konfrontationspolitik bis 1965 ausnutzte. Die Völker Borneos hatten wenige historische Kontakte mit dem südostasiatischen Festland, dafür jedoch um so mehr Beziehungen zu den benachbarten Inseln, und zwar seit Tausenden von Jahren. Auch der Begriff der Minderheiten wird für die Einwohner Borneos besonders fragwürdig, wenn hier manche Stämme für sich allein Riesengebiete bewohnen und sich gar nicht als Minderheiten fühlen können. Wenn aber auch in den malaysischen Teilen Borneos eine Abneigung gegen die befürchtete Malaiisierung besteht, wenn es für die Völker Sabahs und Sarawaks mit ihren alten Kulturen und ihrer oftmals bewiesenen Überlegenheit unmöglich zu sein scheint, sich mit dem Gedanken anzufreunden, daß
"Außenseiter" sie beherrschen sollen (S. 342), so wird doch andererseits mit Recht darauf hingewiesen, daß gerade in Malaysia die Stämme eine Gelegenheit haben, sich mit ihren Anliegen direkt an die Zentralregierung in Kuala Lumpur zu wenden - eine Gelegenheit, die weit über das hinausgeht, was in anderen Ländern der Region möglich ist (S. 337), was aber wohl als unabdingbare Voraussetzung für eine Bereinigung der oftmals noch bestehenden Gegensätze zu gelten hat.

Ein Land, in welchem die Beziehungen der Flachlandbewohner zu den Bergstämmen in jüngster Zeit in ein sehr akutes Stadium getreten sind, ist Thailand (Beiträge von Michael Moerman, Lee W. Huff, F. W. Mote, Hans Manndorff, William R. Geddes, Peter Kandre und Peter Kunstadter). Hier ist das Minderheitenproblem lange Zeit hindurch mit dem Chinesenproblem identifiziert worden (S. 375), so daß man heute in Bangkok wenig über die Sitten und Gebräuche und die Tabus der Bergvölker weiß (S. 479). Auf Grund des geltenden Rechtes ist es für die thailändischen Behörden nicht immer leicht, die Frage zu entscheiden, wer als Thailänder gilt und wer nicht, was Folgen für die Behandlung der Betroffenen und vor allem dafür hat, ob sie an gewissen Vorteilen partizipieren können. Bezeichnend ist auch, daß die thailändische Stammesbevölkerung nie in die Volkszählungen einbezogen wurde. Je angespannter die politischen Verhältnisse an den nördlichen und nordöstlichen sowie an den südlichen Grenzen des Landes jedoch werden, um so mehr bemüht man sich um Verständnis für die Stämme, um Verbesserung des Verhältnisses zu ihnen und um Hebung ihres Lebensstandards. Die verschiedenen, mit entsprechenden Aufgaben betrauten Organisationen werden eingehend dargestellt, und man möchte Kunstadter glauben, wenn er unter Hinweis auf die Toleranz der Thai in kulturellen und religiösen Angelegenheiten die Meinung vertritt, $\mathrm{da} \beta$ es gerade den Thai leichter fallen sollte, bestehende Differenzen auszuräumen (S. 394). 
Unter den Beiträgen über Thailand sind die instruktiven Beschreibungen einzelner Dörfer hervorzuheben, die dem Leser die Probleme des Verhältnisses zwischen Stammesbevölkerung und Regierungsvertretern besser nahebringen als manche theoretische Abhandlung. Besonders herauszustellen ist dabei F. W. Motes Aufsatz über die Siedlungen der erst Anfang der 50er Jahre eingewanderten Yünnan-Chinesen (S. 487 ff.), die zunächst als Tagelöhner arbeiteten, heute aber selbst Arbeiter beschäftigen und mit ihrer erfolgreichen Agrarwirtschaft stimulierend auf ihre Nachbarn wirken. Am Beispiel Thailands ist auch der Einsatz militärischer Einheiten zur Entwicklung der Minderheitengebiete von Interesse (Mobile Development Unit Program), und fast programmatisch ist in diesem Zusammenhang Lee W. Huffs Ansicht: „It is in the interest of all developing nations with sizable military forces actively to seek optimal utilization of them in development roles" (S. 482).

Ein Aspekt, der in besonderer Weise die Komplexität der hier angesprochenen Probleme berührt, ist die Frage des Opiumanbaus. Es ist bekannt, daß einige der wichtigsten Gewinnungsgebiete für Opium im Inneren Südostasiens liegen. Der Opiumanbau ist auch für einige der thailändischen Stämme eine wichtige Existenzgrundlage, deren ersatzloser Entzug katastrophale Folgen haben müßte. Obwohl der Opiumanbau illegal ist, sehen daher die Behörden über den dennoch praktizierten Anbau hinweg. Daß aber die Stämme hierbei einer illegalen Beschäftigung nachgehen, die (gegenwärtig?) gedultet wird, trägt nicht zur Bildung einer Staatsmoral unter den Stämmen bei und bedeutet einen Unsicherheitsfaktor in ihrer Existenz, der ihr Verhältnis $z u$ den Behörden des Landes zusätzlich belasten muß.

Das letzte Land, das in diesem umfangreichen Werk behandelt wird, ist Vietnam (Beiträge von John O'Donnell, Gerald C. Hickey und J. T. McAlister, Jr.), wo der Widerspruch der Minderheitenpolitik des Nordens und des Südens besonders deutlich wird. Während
Nord-Vietnam (ähnlich China) die ethnischen Differenzen anerkennt und durch die Schaffung autonomer Regionen neutralisiert hat, hat man sich in Süd-Vietnam bei zahlreichen politischen Maßnahmen bedenkenlos über die Rechte der Minderheiten hinweggesetzt und durch die Konzentration auf militärische Maßnahmen die vielfältigen Möglichkeiten, zu nationaler Einheit zu kommen, blockiert. Das Projekt der Wehrdörfer, auf das man einst so große Hoffnungen setzte, stellt O'Donnell dar, der uns zeigt, daß - wie so oft und auch anderswo - individuelle Lösungen, die einzig hätten zum Erfolg führen können, nicht gefragt waren und daß letzten Endes Saigon eine gesunde Entwicklung sabotiert hat.

Historische Zusammenhänge vor allem aus der französischen Kolonialzeit zeigt McAlister auf, der im Verhältnis zwischen den "Montagnards" und den Viet Minh den Schlüssel zum Indochina-Krieg sieht, der die französische Herrschaft beendete. Sein Beitrag (S. 771 ff.) faßt noch einmal viele der behandelten Probleme zusammen, und die Schlacht von Dien Bien Phu wird bei ihm zum Symbol der nicht bewältigten Gegensätze in der Minderheitenfrage. Wenn wir von McAlister erfahren, daß die Schlacht von Dien Bien Phu, das in einem bedeutenden Opiumanbaugebiet liegt, eine der größten Konfrontationen ethnischer Gruppierungen in der Geschichte Indochinas war (S. 832) und daß die Kommunisten die Franzosen nicht auf Grund ihrer Ideologie, sondern auf Grund ihrer genauen Kenntnisse über die Berge und Völker Nord-Vietnams besiegten (S. 833), dann glaubt man ihm auch, daß für die Beurteilung der Lage in Südostasien die Beobachtung der Minderheitenpolitik der Chinesen und Vietnamesen viel wichtiger ist als die Beschäftigung mit abstrakten kommunistischen Ideen (S. 835).

Man könnte Kunstadter vorwerfen, daß er eine der grundlegenden ethnischen Beziehungen Südostasiens, nämlich die zu den Überseechinesen, ausgelassen ha- 
be ${ }^{1}$. Das Problem der Úberseechinesen ist jedoch schon in verschiedenen $\mathrm{Pu}$ blikationen behandelt worden, während die Frage der Stämme und ihres Verhältnisses zu den südostasiatischen Regierungen noch weitgehend eine terra incognita ist. Auch der Politikwissenschaftler wie der Staatsrechtler muß sich mit der Lage dieser Stämme beschäftigen, wenn er das heutige Südostasien verstehen will. In diesem Sinne ist das Sammelwerk Kunstadters eine einzigartige Pionierleistung.

Bernhard Großmann

\section{Otto BRaun}

\section{Von Schanghai bis Jänan}

in „horizont", sozialistische Wochenzeitung für internationale Politik und Wirtschaft, Nr. 23-38, 2. Jahrgang (1. Juniheft -3 . Septemberheft 1969).

Ist der Kommunismus in China international oder national? Diese im Westen stets diskutierte Frage ist mit der Spaltung zwischen Peking und Moskau, besonders aber seit dem Ausbruch der chinesischen Kulturrevolution auch im osteuropäischen Block akut geworden. Otto Braun, der jetzige wissenschaftliche Mitarbeiter des Instituts für Gesellschaftswissenschaften beim ZK der SED und ehemalige militärische Berater des Komintern beim ZK der KP Chinas', sucht in seinen Memoiren die "historischen Wurzeln" dieser Frage bloßzulegen, die nach seiner Ansicht bis in die dreißiger, ja zwanziger Jahre zurückreichen.

Nach seiner eigenen Darstellung war Braun von 1932 bis 1939 sieben Jahre lang in China und stand diese ganze Zeit über in enger Verbindung mit der Führung der KP Chinas und der chinesischen Roten Armee. Er hat nicht nur die Bürgerkriegskämpfe in Kiangsi mitgemacht, sondern auch am legendären "Langen Marsch" teilgenommen. Schließlich wirkte er an der kommunistischen
Militärakademie in Jänan. Im Spätsommer 1939 wurde er nach Moskau zurückgerufen.

Der ostdeutsche Autor macht Mao Tsetung vor allem die Bauernrevolution zum Vorwurf und meint, daß der Grundsatz von der führenden Rolle des Proletariats von Mao „subjektiv ernsthaft" in Frage gestellt worden sei. (Forts. 4, Spalte 1). "So sprach er (Mao) häufig vom Proletariat, verstand darunter aber keineswegs die Industriearbeiter, sondern alle Schichten der ärmsten Bevölkerung, Landarbeiter und Halbpächter, Handwerker und Kleinhändler, bis zu Kulis und sogar Bettlern" (Fort. 6, Spalte 3). Es war zwar in der Adresse der Plenartagung des ZK der KP Chinas an den 2. Allchinesischen Sow jetkongreß 1934 in Juichin (Hauptstadt des chinesischen Sowjetgebiets) u. a. auch von der Stärkung und Festigung der führenden Rolle der Arbeiterklasse in allen Organen der chinesischen Sowjetmacht die Rede, doch war diese Formulierung (nach Braun) nur dem Einfluß der „marxistisch-internationalistischen Gruppe", nämlich der Komintern-Anhänger wie $\mathrm{Bo} \mathrm{Ku}$ (Ch'in Panghsien), Lo Fu (Chang Wen-t'ien) u. a. zu verdanken. Das gewisse Kräftegleichgewicht zwischen diesen und den Anhängern Mao Tse-tungs wurde jedoch Anfang 1935 bei der erweiterten Beratung des Politbüros in Tsunyi beendet, als Mao zum Vorsitzenden der Partei gewählt wurde.

„Damit vereinigte Mao nun die Führung von Partei, Regierung und Armee in seiner Hand. Diese Machtkonzentration, erreicht mit Hilfe ihm ergebener Truppenführer, leitete $z$ war nicht sofort, aber im Laufe des nächsten Jahrzehnts zu einer Art Selbstherrschaft Maos über, die ihm die Durchsetzung seiner antimarxistischen und nationalistischen $\mathrm{Li}$ nie ermöglichte."

Alles in allem meint Braun, daß der jetzige "großmachtchauvinistische" Mao damals schon ein „kleinbürgerlicher $\mathrm{Na}$ -

1 Vgl. dazu die Besprechung des Rezensenten in "Verfassung und Redit in Ubersee“, 2. Heft, 1968, S. $230 \mathrm{ff}$.

2 In der übrigen Literatur ist er mit dem dinesischen Namen Li Teh oder Li T'eh bekannt. Vgl. u. a. Charles B. McLane, Soviet Policy and the Chinese Communists 1931-1946, New York 1958, S. 10, $52 \mathrm{f} ., 152 \mathrm{f}$. 